\title{
Exocyst Sec10 Is Involved in Basolateral Protein Translation and Translocation in the Endoplasmic Reticulum
}

\author{
Soo Young Choi ${ }^{\mathrm{a}}$ Ben Fogelgren ${ }^{\mathrm{a}}$ Xiaofeng Zuo ${ }^{\mathrm{a}}$ Liwei Huang ${ }^{\mathrm{a}}$ \\ Sarah McKenna ${ }^{a}$ Vishwanath R. Lingappa ${ }^{c}$ Joshua H. Lipschutz ${ }^{a}$ b \\ ${ }^{a}$ Department of Medicine, University of Pennsylvania, and ${ }^{\text {b }}$ Department of Medicine, Veterans Affairs \\ Medical Center, Philadelphia, Pa., and ' Departments of Physiology and Medicine, University of California, \\ San Francisco, San Francisco, Calif., USA
}

\section{Key Words}

Exocyst $\cdot$ Polarity $\cdot$ Translation $\cdot$ Endoplasmic reticulum

\begin{abstract}
Background: Protein translation and translocation at the rough endoplasmic reticulum (RER) are the first steps in the secretory pathway. The translocon through which newly made proteins are translocated into or across the RER membrane consists of three main subunits: $\operatorname{Sec} 61 \alpha,-\beta$, and $-\gamma$. Sec61 $\beta$ facilitates translocation, and we and others have shown that the highly conserved eight-protein exocyst complex interacts with Sec61 $\beta$. We have also shown that the exocyst is involved in basolateral, not apical, protein synthesis and delivery. Recently, however, exocyst involvement in apical protein delivery has been reported. Furthermore, we have shown that the exocyst is necessary for formation of primary cilia, organelles found on the apical surface. Methods: GST pulldown was performed on lysate of renal tubule cells to investigate biochemical interactions. Cell-free assays consisting of cell-free extracts from rabbit reticulocytes, pancreatic endoplasmic reticulum (ER) microsomal membranes, transcripts of cDNA from apical and basolateral proteins, ATP/GTP, amino acids, and ${ }^{35} \mathrm{~S}$-methionine for protein detection were used to investigate the role of the exocyst in synthesis of polarized
\end{abstract}

proteins. $\mathrm{P}^{32}$-orthophosphate and immunoprecipitation with antibody against Sec61 $\beta$ was used to investigate Sec61 $\beta$ phosphorylation in exocyst Sec10-overexpressing cells. $\boldsymbol{R e}$ sults: Sec10 biochemically interacts with Sec61 $\beta$ using GST pulldown. Using cell-free assays, there is enhanced exocyst recruitment to endoplasmic reticulum membranes following exocyst depletion and basolateral G protein of vesicular stomatitis virus protein translation, compared to apical hemagglutinin of influenza virus protein translation. Finally, Sec10 overexpression increases Sec61 $\beta$ phosphorylation. Conclusion: These data confirm that the exocyst is preferentially involved in basolateral protein translation and translocation, and may well act through the phosphorylation of Sec61 $\beta$.

Copyright $\odot 2012$ S. Karger AG, Basel

\section{Introduction}

Proteins destined for secretion, or delivery to the plasma membrane, are translocated across the rough endoplasmic reticulum (RER) via the translocon in eukaryotic cells. After modification and folding, these proteins are then transported in vesicular carriers to the Golgi complex and trans-Golgi network. From the trans-Golgi network, proteins can be sorted to several

\section{KARGER}

Fax +41613061234 E-Mail karger@karger.ch www.karger.com
(C) 2012 S. Karger AG, Base $1660-2129 / 12 / 1204-0133 \$ 38.00 / 0$

Accessible online at:

www.karger.com/nee
Josh Lipschutz, MD

Renal-Electrolyte and Hypertension Division, Department of Medicine

University of Pennsylvania, Clinical Research Building, Room 538A

415 Curie Blvd., Philadelphia, PA 19104-4539 (USA)

Tel. +1 215573 1848, E-Mail jhlipsch@ mail.med.upenn.edu 
sites, including lysosomes, and to the plasma membrane for insertion or release into the extracellular space [1]. The translocon, or protein-conducting pore in the RER through which newly made proteins are translocated into or across the RER membrane, consists of three main subunits, Sec61 $\alpha,-\beta$, and $-\gamma[2,3]$. While the Sec61 $\alpha$ subunit is primarily responsible for translocation, much less is known about the Sec61 $\beta$ subunit. It is not required for translocation of all proteins in reconstituted liposomes or for viability in yeast, but it does facilitate translocation [4].

Polarized epithelial cells have two distinct plasma membrane domains with very different protein compositions and functions: an apical domain comprising the lumenal surface, and a basolateral domain contacting adjoining and underlying cells. To establish and maintain their polarity, epithelial cells synthesize and then deliver specific proteins to the correct apical or basolateral plasma membrane. There are circumstances where the cell might need to regulate the level of whole classes of proteins, such as those found in the plasma membrane, or in particular in the basolateral plasma membrane of polarized epithelial cells. For example, cells must often alter their shape, polarization, and other characteristics during development and differentiation, and this plasticity could require the coordinate regulation of large classes of proteins. An example of this occurs when MDCK cells are grown in three-dimensional collagen gels, and the cells then form hollow cysts lined by a monolayer of cells with their apical surfaces facing the center. Addition of hepatocyte growth factor causes the cells to first send out long extensions of their basolateral plasma membrane, which requires an increase in basolateral surface area. Eventually, cells migrate out and reorganize into tubules in a process resembling tubulogenesis in vivo [5].

One candidate for a component of the machinery in this process is the exocyst complex, first identified as being involved in the exocytosis of vesicles in yeast [6]. The exocyst is a $750-\mathrm{kDa}$ highly conserved complex comprised of Sec3, Sec5, Sec6, Sec8, Sec10, Sec15, Exo70, and Exo84. Mammalian homologs of all eight exocyst proteins have been identified [7]. In yeast, and most likely mammals, Sec10 and Sec15 act as a bridge between the Rab GTPase Sec4/Rab8, found on the surface of the secretory vesicles carrying polarized proteins, and the rest of the exocyst complex, which is in contact with the plasma membrane [8]. Seb1 is a yeast homolog of Sec61ß. SEB1 was isolated as a multicopy suppressor of the sec15-1 mutation, which, given that Sec15 is a component of the exocyst complex, implied at least a genetic interaction of the exocyst and translocon [9]. In previous reports, we showed that the exocyst interacted with Sec61 $\beta$ in mammalian cells [10], and Toikkanen et al. [11] showed the same thing in yeast. We and others showed that the exocyst has effects on basolateral, but not apical, protein synthesis and delivery $[5,10,12,13]$; however, there was a recent report suggesting that the exocyst is involved in apical protein delivery [14]. We also showed involvement of the exocyst in formation of the primary cilium, an organelle found on the apical surface [15]. These studies prompted us to re-examine the role of the exocyst in basolateral protein synthesis and delivery.

Here, we confirm the interaction between the exocyst and Sec61 $\beta$ and demonstrate, using cell-free assays, that the exocyst indeed preferentially associates with the ER during the synthesis of basolateral, as opposed to apical, membrane proteins. Finally, we show data suggesting that this may be accomplished via phosphorylation of Sec61ß.

\section{Methods}

Cell Culture and Reagents

All MDCK cell lines used were derived from low passage type II MDCK cells that were obtained from Dr. K. Mostov (UCSF, San Francisco, Calif., USA), and which were originally cloned by Dr. D. Louvard (European Molecular Biology Laboratory, Heidelberg, Germany). HEK293 cells were obtained from ATCC (No. CRL-1573).

\section{GST Pulldowns}

Full-length human Sec10 cDNA was cloned in frame into the plasmid pGEX-4T-1 (Amersham Biosciences, Piscataway, N.J., USA), and transformed into the DE3 strain of Escherichia coli (Stratagene, La Jolla, Calif., USA). GST fusion protein expression was induced by adding isopropyl-1-thio- $\beta$-D-galactopyranoside to growing cultures and shaking for an additional $3 \mathrm{~h}$ at $37^{\circ} \mathrm{C}$. Recombinant proteins were purified with Glutathione Sepharose (Amersham Biosciences) following bacterial cell lysis. For pulldown experiments, lysates from wild-type HEK293 cells were incubated overnight with Sec10-GST, or GST only, bound to Glutathione Sepharose. Pulldowns were washed extensively, and then resuspended in Laemmli buffer and boiled, and equal amounts were electrophoresed by SDS-PAGE. Bound Sec61 $\beta$, GAPDH, and Sec 8 were detected by Western blot analysis.

Western Analysis

For pulldowns from HEK293 cell lysates, immunoblotting was performed as previously described [15]. The antibodies used in this study are mouse monoclonal anti-GAPDH (G8795, Sigma, St. Louis, Mo., USA), mouse monoclonal anti-Sec6 and -Sec8 (StressGen), and anti-Sec61ß (made by V.R.L.).

Cell-Free Transcription Linked Translation

Cell-free transcription linked translation was performed essentially as described in [16]. 


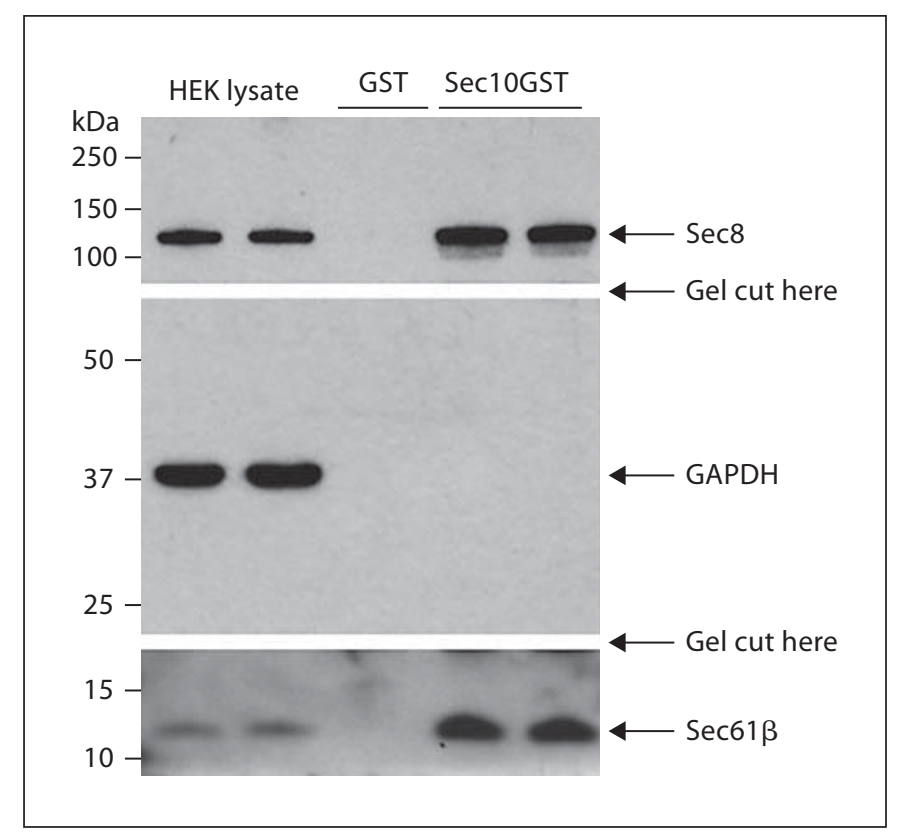

Fig. 1. Sec10 biochemically interacts with Sec61ß. A Sec10-GST fusion protein was expressed in E. coli and purified using Glutathione Sepharose beads. After incubation with HEK293 cell lysate, Sec10-GST, but not GST alone, pulled down Sec61ß. As a positive control for exocyst binding, $\operatorname{Sec} 8$ is shown to bind to Sec10-GST. To demonstrate specificity for the pulldown products, we blotted for GAPDH, a house-keeping protein not known to interact with the exocyst. GAPDH was identified only in cell lysate, and not in pulldown fractions.

\section{Results}

\section{Exocyst Sec10 Biochemically Interacts with Sec61 $\beta$}

We and others previously showed that the exocyst complex interacted with Sec61 $\beta[10,11]$. Here, we confirm a biochemical interaction using a different technique, GST-pulldown. A Sec10-GST fusion protein was purified on Glutathione Sepharose and used as an affinity matrix for the purification of specific binding proteins from human embryonic kidney 293 (HEK293) cell lysate. Sec61 $\beta$ was found in the pulldown fraction and, therefore, identified as a Sec10 binding partner (fig. 1). The Glutathione Sepharose-immobilized Sec10 pulled down exocyst Sec8 from the lysate, serving as a positive control for exocyst binding for the HEK293 lysate. As a negative control, GAPDH, which is not known to interact with the exocyst, was found in the lysate, but was not detected in the pulldown fraction. Bead-immobilized GST alone was also used and no Sec61 $\beta$ protein was detected in the pulldown fraction.

Exocyst in Basolateral Translation
Cell-Free in vitro Assay to Investigate the Role of the Exocyst in Protein Synthesis

Some events, such as translocation, are best studied using cell-free systems. As discussed, in addition to the role of the exocyst in the final stage of the secretory pathway, protein delivery, we and another group showed that the exocyst was involved in the first step of the secretory pathway, protein synthesis, in both yeast and mammalian cells $[10,11]$. The cell-free assay we used here is made up of cell-free extracts from rabbit reticulocytes, canine pancreatic endoplasmic reticulum (ER) microsomal membranes, cDNA from apical and basolateral proteins, ATP/GTP, amino acids, and ${ }^{35} \mathrm{~S}$-methionine for protein detection. ER microsomal membranes can be isolated when cells are disrupted and the ER breaks into fragments and reseals into many small (approx. 100-200 nm diameter) closed vesicles called microsomes, which are relatively easy to purify.

We first confirmed that both the ER microsomal membranes (fig. 2a, lane 1) and the rabbit reticulocyte lysate (fig. 2a, lane 5) contained exocyst proteins. Microsomal membranes were then washed with physiological salts (fig. 2a, lane 2), which had minimal effect, and high salt buffers, which removed the exocyst from the microsomal membranes (fig. 2a, lanes 3 and 4). Exocyst-depleted (salt-washed) microsomal membranes were used in all the translocation experiments.

\section{Translation of Apical and Basolateral Proteins Using the Cell-Free System}

For our studies, we chose two model membrane proteins, the apical hemagglutinin (HA) protein of influenza and the basolateral $\mathrm{G}$ protein of vesicular stomatitis virus (VSVG) [17]. Glycosylation, scored as a shift to higher molecular weight upon addition of ER microsomal membranes (fig. $2 \mathrm{~b}$, lanes 3 and 6 ) and confirmed by a shift down with addition of acceptor tripeptide, a glycosylation inhibitor (fig. 2b, lanes 2 and 5), indicates translocon-dependent insertion into the ER membrane [18].

\section{Increased Exocyst Relocalization Correlates with Increased}

Protein Synthesis during Basolateral Protein Translation

Using the cell-free system, we show a roughly threefold greater amount of exocyst components relocalized from the cytosol to the exocyst-depleted (salt-washed) ER membrane in response to protein synthesis of basolateral protein, VSVG, compared to apical HA (fig. 2c). This correlates with our previous studies where we showed increased basolateral protein synthesis in Sec10-overexpressing versus control MDCK cells $[5,10]$. 


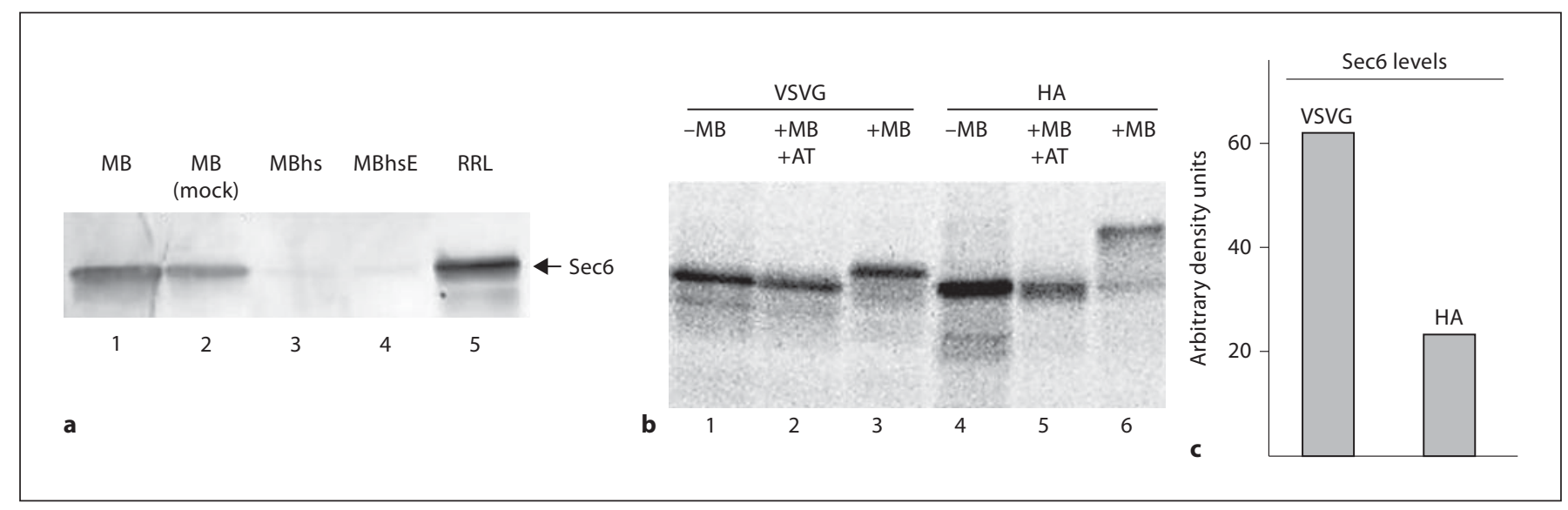

Fig. 2. Apical and basolateral protein translation and translocation. a Salt washes of ER microsomal membranes. $10 \mu \mathrm{l}$ of microsomal membranes (MB) from dog pancreas were washed with physiological salts (mock), high-salt 0.5 M KAc (MBhs), and high salt/EDTA (MBhsE). $2 \mu$ rabbit reticulocyte lysate (RRL) was also analyzed. All lanes were transferred to PVDF membrane and blotted with antibody against the exocyst component Sec6. The exocyst was associated with ER membranes, partly removed by physiological salt wash, completely removed by high-salt wash, and found in RRL. $\mathbf{b}$ Expression of VSVG and HA in the cell-free assay. SP6 expression plasmids were translated in the absence $(-\mathrm{MB})$ or presence $(+\mathrm{MB})$ of ER-derived microsomal membranes, or in the presence of $\mathrm{MB}$ and acceptor tripeptide (AT) as an inhibitor of glycosylation $(+\mathrm{MB} /+\mathrm{AT})$. Glycosylation of VSVG and $\mathrm{HA}$, and hence translocon-dependent insertion into the ER mem- brane, in the presence of microsomal membranes is confirmed by a shift to a higher molecular weight that is reversed upon addition of acceptor tripeptide. c Relocalization of the exocyst from the cytosol to the ER membrane is increased in response to translation of VSVG vs. HA. Transcripts were adjusted to give equivalent levels of expression in the cell-free assay and translated in the presence of identical concentrations of salt-washed (exocyst-depleted) ER microsomal membranes. After the translation reaction was complete, membranes were re-isolated and blotted for the presence of Sec6. Blots were probed with anti-Sec6 antibody (StressGen), followed by alkaline phosphatase-coupled goat antimouse antibody (Promega), developed using Promega Western Blue stabilized substrate for alkaline phosphatase, scanned into Adobe Photoshop, quantified, and graphed as arbitrary density units.

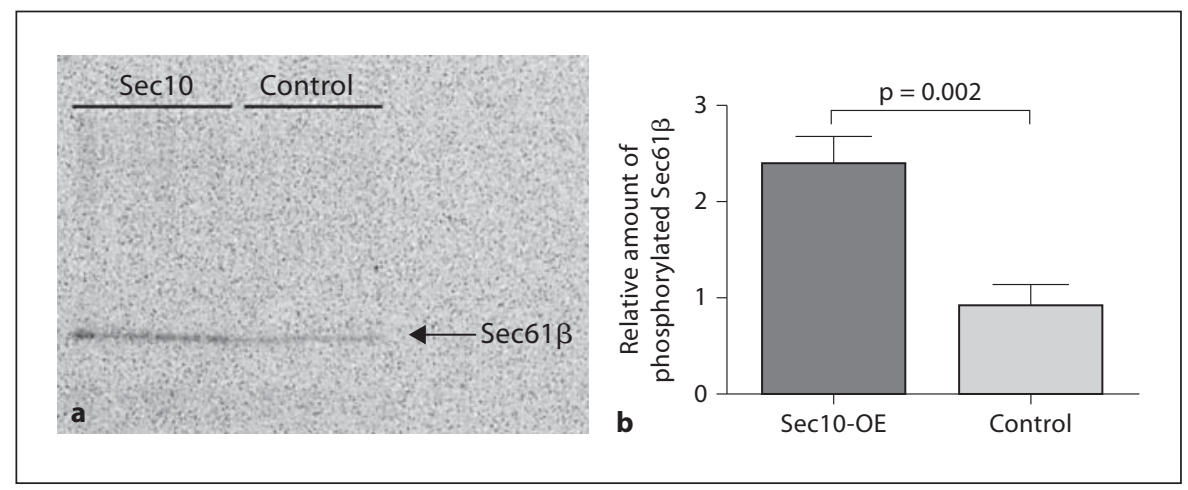

Fig. 3. Exocyst overexpression leads to increased phosphorylation of the translocon component Sec61ß. a Sec10-overexpressing and control MDCK cells were grown on Transwell filters for 7 days and then exposed to $\mathrm{P}^{32}$-orthophosphate. The Sec10-overexpressing and control cells were lysed, equal amounts of protein were confirmed by BCA (a colorimetric assay for total protein quantitation, Pierce), and immunoprecipitation was performed using equal amounts of Sec61 $\beta$ polyclonal antibody. The immunoprecipitate was analyzed by SDS-10\% PAGE and radioactivity was determined using a phosphorimager. $\mathbf{b}$ Quantification of the results from a showed significantly more phosphorylation of Sec61 $\beta$ in Sec10-overexpressing (Sec10-OE) compared to control MDCK cells. This experiment was repeated three times with similar results. 


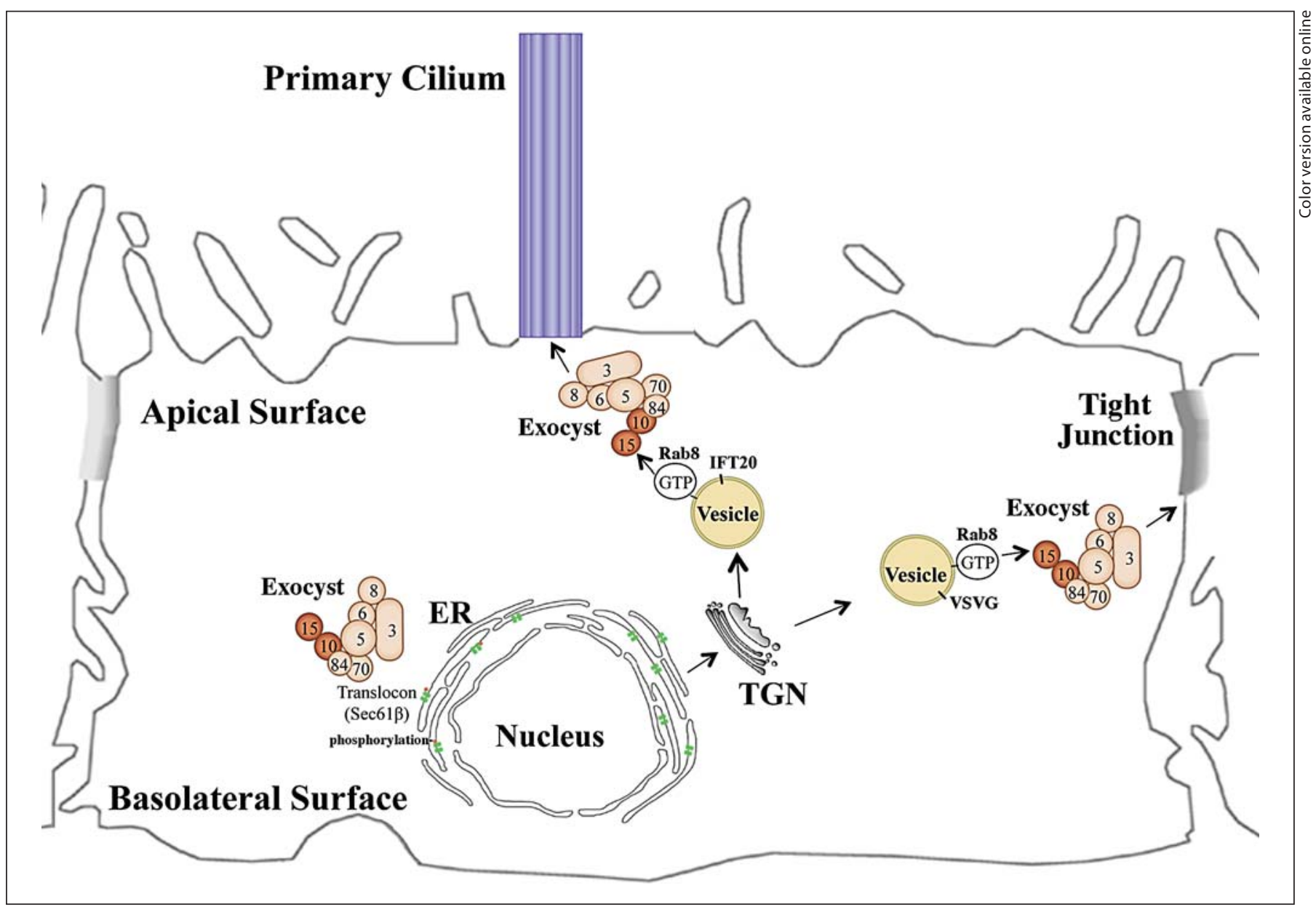

Fig. 4. The role of the exocyst in the secretory pathway. The exocyst complex (comprised of Sec 3, 5, 6, 8, 10, 15, Exo70, and Exo84) acts at the first stage of the secretory pathway, where it regulates the translocon in the ER, most likely by phosphorylation of Sec61 $\beta$, for synthesis of basolateral proteins. The exocyst also acts at the final stages of the secretory pathway to target and dock vesicles carrying basolateral proteins, such as VSVG, at the region just below the tight junction, and ciliary proteins, such as IFT20, at the primary cilium. Sec10 and Sec15 act as a bridge between the Rab GTPase Rab8, found on the surface of the secretory vesicles carrying polarized proteins, and the rest of the exocyst complex, which is in contact with the plasma membrane. The channels in the ER represents the translocon, which includes Sec61 $\beta$, and the small circles in the ER represent the phosphorylation of Sec61ß.

\section{Phosphorylation of Sec61 $\beta$ Is Increased in \\ Sec10-Overexpressing Cells}

It is known that calcium-dependent isoforms of protein kinase $C$ phosphorylate Sec61 $\beta$, causing an increase in the rate of translocation [1]. To start to dissect the mechanism by which Sec10 overexpression increases protein synthesis $[5,10,11]$, we, therefore, examined phosphorylation of Sec61 $\beta$ following exocyst Sec10 overexpression. Using $\mathrm{P}^{32}$ orthophosphate and immunoprecipitation with antibody against Sec61 $\beta$, we found that Sec61 $\beta$ was significantly more phosphorylated in Sec10-overexpressing MDCK cells compared to control MDCK cells (fig. 3).

\section{Discussion}

We report three principal findings, all of which are quite interesting. First, we confirm that the exocyst biochemically interacts with the translocon component Sec61 $\beta$ by using Sec10-GST to pull down Sec61 $\beta$ from HEK293 cell lysate.

Then using a cell-free assay, we demonstrated enhanced recruitment following exocyst depletion and basolateral VSVG protein translation, compared with apical HA protein translation. The advantage of the cell-free in vitro translation/translocation assay over events studied 
'in vivo' (e.g. by pulse chase analysis of cultured mammalian cells) is that cell-free assays are slow and inefficient, yet highly faithful to what occurs in whole cells. As a result, intermediates, and steps not readily detectable in cell culture, are often readily detected in the cell-free assay.

Finally, we show a possible mechanism for the involvement of the exocyst in protein synthesis by demonstrating increased phosphorylation of Sec61 $\beta$ in the presence of Sec10 overexpression. It has previously been shown that the translocon is dynamic and can be regulated [16], and that $\operatorname{Sec} 61 \beta$ is not essential for cotranslational translocation, but does kinetically facilitate it [4]. Gruss et al. [1] showed that calcium-dependent isoforms of protein kinase $\mathrm{C}$ are associated with the RER and phosphorylate essential components of the protein translocation machinery including Sec61 $\beta$. Sec61 $\beta$ is extensively phosphorylated and phosphorylation increases translocation of preprolactin [1]. This becomes especially relevant given that the exocyst has been shown to govern the polarized expression of calcium-signaling complexes and regulation of their activity in the ER of pancreatic acinar cells [19].

These data support our previous results showing that exocyst Sec10, in whole cells, significantly localizes to the ER following overexpression [10], and that Sec10 overexpression results in increased basolateral, but not apical, protein synthesis $[5,10]$. Other groups have also shown specific effects of the exocyst on basolateral, as opposed to apical, proteins $[12,13]$. Recently, however, one report suggested that the exocyst was involved in apical membrane delivery [14]. In addition, we showed that the exocyst localized to and was involved in formation of the primary cilium [15], an organelle found on the apical surface and involved in the pathogenesis of polycystic kidney disease.

For these reasons, we undertook this study and, as discussed, our results confirm that the exocyst interacts with Sec61 $\beta$ and preferentially participates in the synthesis and delivery of basolateral, as opposed to apical, membrane proteins. Our recent study demonstrating that the exocyst is necessary for ciliogenesis adds to the growing body of evidence that primary cilia, while found on the apical surface, are not true 'apical organelles' [15]. This may explain the fact that primary cilia contain many proteins that localize to the basolateral membrane, including polycystins- 1 and -2 , and fibrocystin [20]. Rab8, a small GTPase that is homologous to yeast Sec4, is a candidate for regulating the exocyst at primary cilia [21], and Rab8 is also involved in basolateral trafficking and has been shown to be necessary for ciliogenesis [22]. Ciliary proteins, such as the kinesin-2 motor KIF17, have also been shown to contain a nuclear localization signal and utilize nuclear import proteins, such as importin- $\beta 2$, to gain entry into the primary cilium [23]. Furthermore, the primary cilium contains a highly condensed bilayer domain at its base [24], and it was recently shown that septin 2 formed a diffusion barrier separating ciliary membrane from the surrounding apical membrane [25], providing additional support for the idea that the primary cilium is a distinct cellular organelle.

In summary, because the exocyst has effects on other cellular processes besides protein synthesis (e.g. protein delivery), we used cell-free studies, performed in a 'minimal' setting in which other cellular processes were largely absent, to show that the exocyst preferentially participates in basolateral protein synthesis in the ER, and may well act through the phosphorylation of Sec61ß. The fact that the exocyst is involved in the first stage of the secretory pathway, protein synthesis and translocation, as we show here, in addition to its well-described role in targeting and docking vesicles in the final stages of the secretory pathway [8] (see fig. 4), raises the intriguing possibility that the exocyst could be centrally involved in regulating the entire secretory pathway.

\section{Acknowledgements}

This work was supported in part by grants from the VA (Merit Award to J.H.L.), NIH (DK069909 and DK047757 to J.H.L.; K01DK087852 to B.F., K08DK093625 to L.H.), University of Pennsylvania Research Foundation (to J.H.L.), and Satellite Healthcare (Norman S. Coplon Extramural Research Grant to J.H.L.).

\section{Disclosure Statement}

V.R.L. is a founder and officer of Prosetta Inc.

References

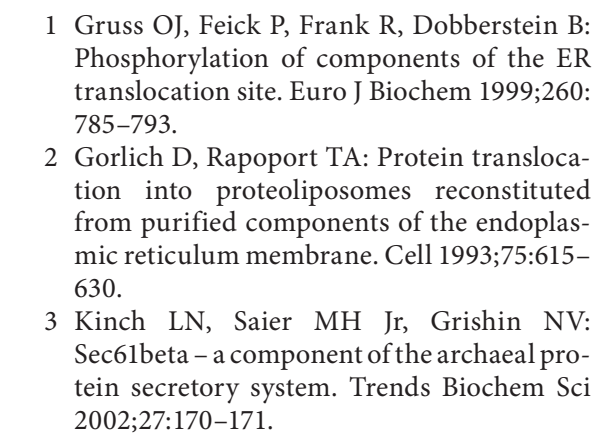

Choi/Fogelgren/Zuo/Huang/McKenna/ Lingappa/Lipschutz 
-4 Kalies KU, Gorlich D, Rapoport TA: Binding of ribosomes to the rough endoplasmic reticulum mediated by the Sec61p-complex. J Cell Biol 1994;126:925-934.

5 Lipschutz JH, Guo W, O’Brien LE, Nguyen $\mathrm{YH}$, Novick P, Mostov KE: Exocyst is involved in cystogenesis and tubulogenesis and acts by modulating synthesis and delivery of basolateral plasma membrane and secretory proteins. Mol Biol Cell 2000;11:4259-4275.

6 Novick P, Field C, Schekman R: Identification of 23 complementation groups required for post-translational events in the yeast secretory pathway. Cell 1980;21:205-221.

$\checkmark 7$ Hsu S, Ting AE, Hazuka CD, Davanger S, Kenny JW, Kee Y, Scheller RH: The mammalian brain rsec6/8 complex. Neuron 1996;17: 1209-1219.

8 Lipschutz JH, Mostov KE: The many masters of the exocyst. Curr Biol 2002;12:R212R214.

9 Toikkanen J, Gatti E, Takei K, Saloheimo M, Olkkonen VM, Soderlund H, De Camilli P, Keranen S: Yeast protein translocation complex: isolation of two genes SEB1 and SEB2 encoding proteins homologous to the Sec61 beta subunit. Yeast 1996;12:425-438.

10 Lipschutz JH, Lingappa VR, Mostov KE: The exocyst affects protein synthesis by acting on the translocation machinery of the endoplasmic reticulum. J Biol Chem 2003;278:2095420960.

-11 Toikkanen JH, Miller KJ, Soderlund H, Jantti J, Keranen S: The beta subunit of the Sec61p ER translocon interacts with the exocyst complex in Saccharomyces cerevisiae. J Biol Chem 2003;278:20946-20953.
12 Grindstaff KK, Yeaman C, Anandasabapathy N, Hsu S, Rodriguez-Boulan R, Scheller $\mathrm{RH}$, Nelson WJ: Sec6/8 complex is recruited to cell-cell contacts and specifies transport vesicle delivery to the basal-lateral membrane in epithelial cells. Cell 1998;93:731740.

13 Moskalenko S, Henry DO, Rosse C, Mirey G, Camonis JH, White MA: The exocyst is a Ral effector complex. Nat Cell Biol 2002;4:6672 .

14 Oztan A, Silvis M, Weisz OA, Bradbury NA Hsu SC, Goldenring JR, Yeaman C, Apodaca $\mathrm{G}$ : Exocyst requirement for endocytic traffic directed toward the apical and basolateral poles of polarized MDCK cells. Mol Biol Cell 2007;18:3978-3992.

15 Zuo X, Guo W, Lipschutz JH: The exocyst protein Sec10 is necessary for primary ciliogenesis and cystogenesis in vitro. Mol Biol Cell 2009;20:2522-2529.

16 Hegde RS, Lingappa VR: Sequence-specific alteration of the ribosome-membrane junction exposes nascent secretory proteins to the cytosol. Cell 1996;85:217-228.

17 Le Bivic A, Real FX, Rodriguez-Boulan E. Vectorial targeting of apical and basolateral plasma membrane proteins in a human adenocarcinoma epithelial cell line. Proc Natl Acad Sci USA 1989;86:9313-9317.

18 Lingappa VR, Katz FN, Lodish HF, Blobel G: A signal sequence for the insertion of a transmembrane glycoprotein. Similarities to the signals of secretory proteins in primary structure and function. J Biol Chem 1978; 253:8667-8670.

19 Shin DM, Zhao XS, Zeng W, Mozhayeva M, Muallem S: The mammalian Sec6/8 complex interacts with $\mathrm{Ca}(2+)$ signaling complexes and regulates their activity. J Cell Biol 2000 150:1101-1112.
20 Wang S, Zhang J, Nauli SM, Li X, Starremans PG, Luo Y, Roberts KA, Zhou J: Fibrocystin/ polyductin, found in the same protein complex with polycystin-2, regulates calcium responses in kidney epithelia. Mol Cell Biol 2007;27:3241-3252.

-21 Knodler A, Feng S, Zhang J, Zhang X, Das A, Peranen J, Guo W: Coordination of Rab8 and Rab11 in primary ciliogenesis. Proc Natl Acad Sci USA 2010;107:6346-6351.

-22 Nachury MV, Loktev AV, Zhang Q, Westlake CJ, Peranen J, Merdes A, Slusarski DC, Scheller RH, Bazan JF, Sheffield VC, Jackson PK: A core complex of BBS proteins cooperates with the GTPase Rab8 to promote ciliary membrane biogenesis. Cell 2007;129:12011213.

-23 Dishinger JF, Kee HL, Jenkins PM, Fan S, Hurd TW, Hammond JW, Truong YN, Margolis B, Martens JR, Verhey KJ: Ciliary entry of the kinesin-2 motor KIF17 is regulated by importin- $\beta 2$ and Ran-GTP. Nat Cell Biol 2010;12:703-710.

24 Vieira OV, Gaus K, Verkade P, Fullekrug J, Vaz WL, Simons K: FAPP2, cilium formation, and compartmentalization of the apical membrane in polarized Madin-Darby canine kidney (MDCK) cells. Proc Natl Acad Sci USA 2006;103:18556-18561.

$\checkmark 25 \mathrm{Hu}$ Q, Milenkovic L, Jin H, Scott MP, Nachury MV, Spiliotis ET, Nelson WJ: A septin diffusion barrier at the base of the primary cilium maintains ciliary membrane protein distribution. Science 2010;329:436-439. 